\title{
PARTIAL REGULARITY AND $t$-ANALYTIC SETS FOR BANACH FUNCTION ALGEBRAS
}

\author{
JOEL FEINSTEIN AND RAYMOND MORTINI
}

\begin{abstract}
In this note we introduce the notion of $t$-analytic sets. Using this concept, we construct a class of closed prime ideals in Banach function algebras and discuss some problems related to Alling's conjecture in $H^{\infty}$. A description of all closed $t$-analytic sets for the disk-algebra is given. Moreover, we show that some of the assertions in [8] concerning the $O$-analyticity and $S$-regularity of certain Banach function algebras are not correct. We also determine the largest set on which a Douglas algebra is pointwise regular.
\end{abstract}

2.9.2010

\section{INTRODUCTION}

In this note we will show that some refinements of notions appearing in a paper by Daoui, Mahzouli and Zerouali [8] in connection with local/restricted decomposability of multiplication operators on commutative, semisimple Banach algebras have a close connection with Alling's conjecture on the structure of closed prime ideals in $H^{\infty}$.

Let $X$ be a compact Hausdorff space. We denote by $C(X)$ the algebra of all continuous, complex-valued functions on $X$. With respect to the uniform norm, $\|\cdot\|_{X}$, on $X, C(X)$ is a unital, commutative, semisimple Banach algebra.

Let $A$ be a commutative, unital Banach algebra over the field of complex numbers $\mathbb{C}$. The character space (or spectrum), $M(A)$, of $A$ is the set of all non-zero multiplicative linear functionals on $A$. When endowed with the usual Gelfand topology (the relative weak-*-topology as a subset of $A^{*}$ ), $M(A)$ becomes a compact Hausdorff space. Using Gelfand theory, we may identify $M(A)$ with the maximal ideal space of $A$. In the case where $A$ is semisimple, $A$ is isomorphic to the algebra $\hat{A} \subseteq C(M(A))$ consisting of the set of Gelfand transforms $\hat{f}: m \mapsto m(f)$ of elements $f \in A$. In this case, identifying $f$ with $\hat{f}$, we may regard $A$ as a Banach function algebra on $M(A)$. With this identification, $\|f\|_{M(A)}$ is equal to the spectral radius of $f$ in the algebra $A$. If the spectral radius and the norm on $A$ are equal for all $f \in A$, then $A$ is a uniform algebra on $M(A)$.

It is useful to remember the result that a commutative, unital Banach algebra $A$ is a uniform algebra if and only if $\left\|f^{2}\right\|=\|f\|^{2}$ for all $f \in A$. Finally, the Shilov boundary, $\partial A$, of $A$ is the smallest closed subset of $M(A)$ on which every $f \in A$ achieves its maximum modulus.

2010 Mathematics Subject Classification. Primary 46J15 ; Secondary 30H50.

Key words and phrases. Banach function algebras; regularity points; $t$-analytic sets; closed prime ideals; bounded analytic functions; Douglas algebras; Alling's conjecture. 
We refer the reader to the books of Dales [7] (especially Chapter 4), Browder [2], Gamelin [11], and Leibowitz [24] for various aspects of the theory of commutative Banach algebras.

Throughout this note, we will work only with semi-simple, unital, commutative Banach algebras, and we look upon them as Banach function algebras defined on their compact spectrum $M(A)$.

Some of our results are valid for more general algebras $A$ of complex-valued functions defined on sets other than $M(A)$, or for commutative Banach algebras which are not semisimple. However, these more general algebras are not our primary concern. For the sake of clarity, we will not attempt to state our results in full generality.

\section{Partial Regularity and $t$-Analyticity: the Definitions}

2.1. Regularity of type I and type II. First we recall the definitions of regularity and normality for Banach function algebras.

Definition 2.1. Let $A$ be a Banach function algebra.

(1) $A$ is regular if, for all $x \in M(A)$ and all closed sets $E \subseteq M(A) \backslash\{x\}$, there exists $f \in A$ such that $f \equiv 1$ on $E$ and $f(x)=0$;

(2) $A$ is normal if, for each pair of disjoint closed sets $E$ and $F$ in $M(A)$ there exists $f \in A$ such that $f \equiv 1$ on $E$ and $f \equiv 0$ on $F$.

It is standard that regularity and normality are equivalent (see [7, Proposition 4.1.18]). We next recall the definitions of the hull of an ideal and the kernel of a set, in order to define the hull-kernel topology on $M(A)$.

Definition 2.2. Let $A$ be a Banach function algebra. For $f \in A$, the zero set of $f$ is the closed set $\{x \in M(A): f(x)=0\}$, and is denoted by $Z_{A}(f)$, or by $Z(f)$ if the algebra under consideration is clear. Similarly, the hull (or zero set) of an ideal $J$ in $A$ is the closed set $\bigcap_{f \in J} Z_{A}(f)$, and is denoted by $Z_{A}(J)$, or $Z(J)$.

Let $E \subseteq M(A)$. Then the kernel of $E$ is the closed ideal

$$
\{f \in A: f \equiv 0 \text { on } E\},
$$

and is denoted by $I_{A}(E)$ or $I(E)$.

Provided that there is no ambiguity in the algebra $A$ under consideration, we denote by $\hat{E}$ the hull of $I(E)$; that is $\hat{E}=Z(I(E))$. The hull-kernel topology on $M(A)$ is the topology whose closed sets are those sets $E \subseteq M(A)$ such that $E=\hat{E}$.

It is standard that the hull-kernel topology is a compact topology on $M(A)$ which is weaker than the Gelfand topology, and (hence) that these two topologies coincide if and only if the hull-kernel topology is Hausdorff. This occurs if and only if the Banach function algebra $A$ is regular (and hence normal). The hullkernel topology and regularity are also closely related to the decomposability of multiplication operators on $A$, as discussed in [27] (see also [6, 10, 8, 23]).

For each ideal $J$ in $A$, we have that $Z(J)$ is, in fact, hull-kernel closed. Thus the hull-kernel closed subsets of $M(A)$ are precisely the hulls of ideals in $A$. Moreover, for each $f \in A$, the hull of the principal ideal $f A$ is simply $Z(f)$, and so the zero sets of functions in $A$ are hull-kernel closed. In fact, the collection of 'co-zero sets' $\{M(A) \backslash Z(f): f \in A\}$ is a base for the hull-kernel topology.

We now consider the identity map on $M(A), \operatorname{Id}_{M(A)}$, regarded as a map from $M(A)$ with the hull-kernel topology to $M(A)$ with the Gelfand topology. It follows 
from the previous paragraphs that this map is continuous if and only if $A$ is regular. In [9], in order to discuss a localized (point by point) version of the connection between the hull-kernel topology and regularity, the points at which this map is continuous were investigated.

Definition 2.3. Let $A$ be a Banach function algebra. For each $f \in A$,

$$
\operatorname{supp} f:=\overline{\{x \in M(A): f(x) \neq 0\}}
$$

is the (closed) support of $f$.

(1) A point $x \in M(A)$ is called a regularity point of type one for $A$, denoted by $x \in \mathscr{R}_{I}$, if for every neighborhood $V$ of $x$ in $M(A)$, there exists a function $f \in A$ such that $f(x)=1$ and the support of $f$ is contained in $V$.

(2) Let $X \subseteq M(A)$. Then we say that $A$ is pointwise regular on $X$, if every $x \in X$ is a regularity point of type one for $A$. We also say in that case that $X$ is a set of regularity (of) type one for $A$.

It is clear that $A$ is regular if and only if $M(A)$ is a set of regularity type one for $A$. Later on, in Section 3, we will determine for special algebras the largest set $X_{\max }$ in $M(A)$ on which $A$ is pointwise regular. That set may be empty, though. This is the case, for example, for the disk-algebra $A(\overline{\mathbb{D}})$. If $X$ contains the Shilov boundary of $A$ and if $A$ is pointwise regular on $X$, then $\left.A\right|_{X}$ is a 'regular function algebra on $X^{\prime}$ in the sense as defined for example in $[7$, p. 412] or $[19$, p. 189]. We do not know whether the converse holds.

It follows from the results in [9] that the points in $\mathscr{R}_{I}$ are exactly those points $x \in M(A)$ for which the identity map $\operatorname{Id}_{M(A)}$ is continuous at $x$ in the sense described above [9, Lemma 2.1]. Hence we may say that $x$ is a regularity point of type one if and only if $x$ is an hk-continuity point for $\operatorname{Id}_{M(A)}$, (or briefly called a 'point of continuity for $A$ ' in [9]).

It is also known that $x \in M(A)$ is an $h k$-continuity point for $\operatorname{Id}_{M(A)}$ if and only if every function in $A$ is continuous at $x$ when $M(A)$ is given the hull-kernel topology (see [9]). Of course, the set of all $h k$-continuity points for $A$ is the maximum $h k$-continuity set for $A$. For completeness, let us say that $x \in M(A)$ is an $h k$ discontinuity point, if it is not an $h k$-continuity point.

Note also that if $S=M(A) \backslash X$, where $X \subseteq M(A)$, then our notion of $X$ being a set of regularity type one for $A$ is equivalent to the notion of $S$-regularity of $A$ given in [8].

Finally, we note that any regularity point of type one (or equivalently any $h k$ continuity point for $A$ ) is necessarily contained in the Shilov boundary $\partial A$ of $A$. This fact, which is stated near the top of page 57 of [9], is almost immediate from the definitions.

So this gives us one of many possible notions of 'partial regularity' for $A$.

We continue this section by giving some related partial regularity conditions involving $k$-hulls, as introduced in [15] in order to study spectral synthesis sets in $H^{\infty}+C$; see also [17].

Definition 2.4. Let $A$ be a Banach function algebra.

(1) For $x \in M(A)$, let $J_{A}(x)$ (or simply $J(x)$ if the algebra $A$ is unambiguous) be the ideal of those functions in $A$ that vanish (within $M(A)$ ) in a neighborhood of $x$. The hull of $J_{A}(x)$ is denoted by $k_{A}(x)$ (or $k(x)$ ), and is called the $k$-hull of $x$. 
(2) A point $x \in M(A)$ is called a regularity point of type two, denoted by $x \in \mathscr{R}_{I I}$, if $k_{A}(x)=\{x\}$.

Note that in [9], the elements $x \in \mathscr{R}_{I I}$ were called $R$-points.

For the readers convenience, we present a short proof that $A$ is regular if and only if each $x \in M(A)$ is a regularity point of type two.

Proposition 2.1. Let $A$ be a Banach function algebra.Then $A$ is regular if and only if $k_{A}(x)=\{x\}$ for every $x \in M(A)$.

Proof. Suppose that $A$ is regular and let $x, y \in M(A)$ with $x \neq y$. As mentioned, $A$ actually is a normal algebra; that is for any two disjoint closed sets $E$ and $F$ in $M(A)$ there exists $f \in A$ with $f \equiv 0$ on $E$ and $f \equiv 1$ on $F$. Now by taking for $E$ a closed neighborhood of $x$ with $y \notin E$ and setting $F=\{y\}$, we obtain a function $f \in J_{A}(x)$ with $f(y) \neq 0$. So $k_{A}(x)=\{x\}$.

Now suppose that $k_{A}(x)=\{x\}$ for every $x \in M(A)$. Let $m \in M(A)$ and $E$ a compact subset of $M(A) \backslash\{m\}$. Then, for each $y \in E$, there exists $f_{y} \in J_{A}(y)$ with $f_{y}(m)=1$. A standard compactness argument then yields the desired function $f=f_{y_{1}} \ldots f_{y_{n}} \in A$ with $f \equiv 0$ on $E$ and $f(m)=1$. Thus $m$ is a regularity point of type one and so $A$ is regular.

The properties of $R$-points and $h k$-continuity points were discussed in detail in [9]. If $x$ is not an $R$-point for $A$, then the points of $k(x) \backslash\{x\}$ are points of $h k$ discontinuity for $A$. Similarly, $x$ is a point of $h k$-discontinuity for $A$ if and only if there is at least one point $y \in M(A) \backslash\{x\}$ with $x \in k(y)$; each such point $y$ is then a non- $R$-point for $A$. Another way to say this is that $x$ is a point of $h k$-continuity for $A$ if and only if there are no points $y \in M(A) \backslash\{x\}$ with $x \in k(y)$. In general, though, there are no inclusion relations between the set $\mathscr{R}_{I}$ of $h k$-continuity points and the set $\mathscr{R}_{I I}$ of $R$-points.

2.2. $t$-analyticity. We now define the notion of $t$-analyticity for subsets of $M(A)$, a notion which is in some sense opposite to the notions of sets of regularity type one or two. It incorporates the fact that some trace of analyticity remains when the functions are restricted to a given set, essentially the identity principle/quasianalyticity for the restrictions of the functions.

Definition 2.5. Let $A$ be a Banach function algebra,and let $E \subseteq M(A)$. We say that $E$ is a $t$-analytic set for $A$ (or simply ' $E$ is $t$-analytic' if the algebra $A$ is clear), if for every $f \in A$ and every relatively open, nonvoid subset $U$ in $E$ one has that $f \equiv 0$ on $U$ implies that $f \equiv 0$ on $E$. We say that $E$ is componentwise t-analytic (for $A$ ) if every connected component of $E$ is a $t$-analytic set.

The most obvious $t$-analytic sets are singletons and the empty set.

In [8] the authors only consider the case where $E$ is an open set in $M(A)$. They say that a Banach function algebra $A$ is $O$-analytic if $O \subseteq M(A)$ is an open $t$ analytic set for $A$. They claim and attempt to prove that there exists a maximum open set $O$ for which $A$ is $O$-analytic. However this claim and its proof are incorrect. We give the following easy example to demonstrate this. This example also shows that a union of open $t$-analytic sets need not be $t$-analytic, contrary to an implicit assumption made in [8]

Let $X$ be a (non-empty) compact set in $\mathbb{C}$ and $X^{\circ}$ its interior. Then $A(X)$ denotes the uniform algebra $\left\{f \in C(X): f\right.$ is analytic on $\left.X^{\circ}\right\}$. By a theorem 
of Arens (see [7, Theorem 4.3.14] or [11, Chapter II, Theorem 1.9]), we have $M(A(X))=X$. Now consider the closed disks $O_{1}=\{z \in \mathbb{C}:|z| \leq 1\}$ and $O_{2}=\{z \in \mathbb{C}:|z-3| \leq 1\}$. Set $X=O_{1} \cup O_{2}$, and let $A$ be the uniform algebra $A(X)$. Then $M(A)=X$, and the sets $O_{1}$ and $O_{2}$ are open $t$-analytic sets for $A$, but $O_{1} \cup O_{2}$ is clearly not a $t$-analytic set for $A$. Moreover, no $t$-analytic set for $A$ can meet both $O_{1}$ and $O_{2}$. Thus there is no maximum open $t$-analytic set for $A$ in this case.

All the subsequent "results" in [8] that use this incorrect claim must be adapted appropriately.

Note, however, that it is true that, whenever $O_{1}$ and $O_{2}$ are open $t$-analytic sets for $A$ such that $O_{1} \cap O_{2} \neq \emptyset$, then $O_{1} \cup O_{2}$ is also an open $t$-analytic set for $A$.

Lemma 2.2. Let $A$ be a Banach function algebra and $C \subseteq M(A)$ a connected open set. Suppose that $C$ is a union of open sets $C_{\lambda}$ which are $t$-analytic for $A$. Then $C$ is t-analytic for $A$.

Proof. Let $U$ be an open set in $M(A)$ satisfying $U \cap C \neq \emptyset$. Suppose that $f \in A$ vanishes identically on $U \cap C$. We need to show that $f \equiv 0$ on $C$.

By our assumption, there is some $C_{\lambda_{0}}$ such that $U \cap C_{\lambda_{0}} \neq \emptyset$. Since $C_{\lambda_{0}}$ is $t$-analytic, $f \equiv 0$ on $C_{\lambda_{0}}$. Now suppose to the contrary that $f$ does not vanish identically on every $C_{\mu}$. Let $\mathfrak{C}_{1}$ be the union of all those $C_{\mu}$ such $f \equiv 0$ on $C_{\mu}$ and let $\mathfrak{C}_{2}$ be the union of the remaining ones. Note that by our hypothesis $\mathfrak{C}_{1}$ and $\mathfrak{C}_{2}$ are nonvoid open sets.

Since $C$ is connected, $\mathfrak{C}_{1} \cap \mathfrak{C}_{2} \neq \emptyset$. Now $f \equiv 0$ on the open set $\mathfrak{C}_{1}$. Let $\mu_{0}$ be chosen so that $C_{\mu_{0}} \subseteq \mathfrak{C}_{2}$ and $C_{\mu_{0}} \cap \mathfrak{C}_{1} \neq \emptyset$. The $t$-analyticity of $C_{\mu_{0}}$ now implies that $f \equiv 0$ on $C_{\mu_{0}}$. This is a contradiction. Thus $\mathfrak{C}_{2}=\emptyset$ (since $C_{\lambda_{0}} \subseteq \mathfrak{C}_{1} \neq \emptyset$ ), and so $f \equiv 0$ on $C$. Hence $C$ is $t$-analytic.

Proposition 2.3. Let $A$ be a Banach function algebra, and let $\mathcal{T}$ be the set of all those open subsets $U$ of $M(A)$ such that all of the connected components of $U$ are also open in $M(A)$. Set

$$
\mathcal{S}=\{U \in \mathcal{T}: U \text { is componentwise t-analytic for } A\} .
$$

Then $\bigcup_{U \in \mathcal{S}} U$ is also in $\mathcal{S}$, and is thus the maximum element of $\mathcal{S}$.

Proof. Let $V=\bigcup_{U \in \mathcal{S}} U$. We first show that $V \in \mathcal{T}$. Obviously $V$ is open. Let $C$ be a connected component of $V$. Write $U=\bigcup_{\lambda \in \Lambda_{U}} C_{\lambda}^{U}$, where $C_{\lambda}^{U}$ is a connected component of $U$. Then

$$
V=\bigcup_{U \in \mathcal{S}} \bigcup_{\lambda \in \Lambda_{U}} C_{\lambda}^{U}
$$

Since $V$ is a union of connected open sets, it follows that the component $C$ of $V$ itself is the union of all those $C_{\lambda}^{U}$ that are entirely contained in $C$. All these $C_{\lambda}^{U}$ are open; hence $C$ is open. Thus $V \in \mathcal{T}$.

Next we show that $V \in \mathcal{S}$. Again, let $C$ be a connected component of $V$. In the representation for $C$ above, all the $C_{\lambda}^{U}$ are open and $t$-analytic for $A$; hence, by Lemma $2.2, C$ is $t$-analytic.

In particular, if $M(A)$ is locally connected, then $\mathcal{T}$ is simply the whole of the topology on $M(A)$, and we see that the set $V$ is the maximum, open, componentwise $t$-analytic set for $A$. However, if $M(A)$ is not locally connected, there may be a shortage of connected open sets. 
For example, the spectrum of $H^{\infty}$ is not locally connected. This follows from the fact that for interpolating Blaschke products $b$ with infinitely many zeros and $\varepsilon>0$ small, the intersection of the level set $\left\{x \in M\left(H^{\infty}\right):|b(x)|<\varepsilon\right\}$ of $b$ with $\mathbb{D}$ is a disjoint union of infinitely many components (see [20]).

In general, when $E_{1}$ and $E_{2}$ are $t$-analytic sets for $A$ with $E_{1} \cap E_{2} \neq \emptyset$, it is not necessarily the case that $E_{1} \cup E_{2}$ is a $t$-analytic set for $A$. Nor is it necessarily the case, even when $M(A)$ is locally connected, that there is a maximum, componentwise $t$-analytic set for $A$. To illustrate this, consider the following easy example, similar to our example above.

Example 2.4. Consider the closed disks $X_{1}=\{z \in \mathbb{C}:|z| \leq 1\}$ and $X_{2}$ $=\{z \in \mathbb{C}:|z-2| \leq 1\}$. Set $X=X_{1} \cup X_{2}$, and let $A$ be the uniform algebra $A(X)$. Then $M(A)=X$, which is locally connected, and the sets $X_{1}$ and $X_{2}$ are (non-open) $t$-analytic sets for $A$ with $X_{1} \cap X_{2}=\{1\} \neq \emptyset$. However, $X=X_{1} \cup X_{2}$ is not $t$-analytic. For example, the function which is constantly zero on $X_{2}$ but equal to $z-1$ on $X_{1}$ is a function in $A$ which is identically zero on a non-empty open subset of $X$, but which is not constant on $X$.

Since $X_{1}, X_{2}$ and $X$ are connected, it follows that there is no maximum, componentwise $t$-analytic set for $A$ in this case.

2.3. Scheme of the paper. In [8] the existence of a smallest set $S_{A}$ for which $A$ is $S_{A}$-regular is shown. In view of our earlier comments, $S_{A}$ is, in fact, equal to the set of all $h k$-discontinuity points for $A$, and so this is the complement of the maximum $h k$-continuity set for $A$. It is claimed in [8] that for Douglas algebras $D$, the set $S_{D}$ is equal to the union of the support sets of all the representing measures. This is not correct. In fact, we will show in Section 3 that $S_{D}$ equals $M(D) \backslash \partial D$, where $\partial D$ is the Shilov boundary of $D$. Also, their determination of the largest open set $O_{D}$ for which $D$ is $O_{D}$-analytic is not correct either. They appear to have been under the impression that the support sets (on $\partial D$ ) of the representing measures $\mu_{m}$ for points $m \in M(D)$ have non-void interior. This is not correct. We will show in Section 5 that for many, but not all, Douglas algebras, including $H^{\infty}+C$, there is no non-empty open $t$-analytic set $O \subseteq M(D)$. In this section we will also discuss the general structure of $t$-analytic sets in Douglas algebras, with emphasis on the algebra $H^{\infty}+C$. For certain points $x \in M\left(H^{\infty}+C\right)$ we will explicitly determine the maximal $t$-analytic sets containing $x$.

This will be preceded in Section 4 by the proof of the fact that every $t$-analytic set for a Banach function algebra $A$ is contained in a maximal $t$-analytic set. These maximal $t$-analytic sets will automatically be closed. We will also show that, apart from singletons, the $t$-analytic sets for $A$ do not contian any regularity points of type I or II.

A complete characterization of the closed $t$-analytic sets for the disk-algebra is given in Section 4, too.

This section also contains one of our main results: namely that each $t$-analytic set induces a closed prime ideal in $A$. Alling's conjecture tells us that in $H^{\infty}$ every non-maximal closed prime ideal coincides with the set of functions vanishing identically on a nontrivial Gleason part. In the final section, Section 6, we will discuss the relations between Gleason parts, the hulls of closed prime ideals and the $t$-analytic sets and address the question whether there are any counterexamples to Alling's conjecture. 


\section{Partial Regularity for Douglas algebras}

As usual $\mathbb{T}$ denotes the unit circle $\{z \in \mathbb{C}:|z|=1\}$. Let $L^{\infty}(\mathbb{T})$ be the space of (equivalence classes) of measurable and essentially bounded functions on $\mathbb{T}$, endowed with the essential supremum norm on $\mathbb{T}$. Moreover, let $H^{\infty}(\mathbb{T})$ be the subalgebra of all those functions in $L^{\infty}(\mathbb{T})$ whose negative Fourier coefficients vanish. It is well known that, with respect to the essential supremum norm on $\mathbb{T}, H^{\infty}(\mathbb{T})$ is isometrically isomorphic to the uniform algebra $H^{\infty}$ of all bounded analytic functions in $\mathbb{D}$. A closed subalgebra of $L^{\infty}(\mathbb{T})$ that strictly contains $H^{\infty}(\mathbb{T})$ is called a Douglas algebra.

The smallest Douglas algebra is $H^{\infty}(\mathbb{T})+C(\mathbb{T})$, which we denote for short by $H^{\infty}+C$. Here $C(\mathbb{T})$ is the set of all complex-valued continuous functions on $\mathbb{T}$. The exact structure of these algebras is given in the celebrated Chang-Marshall theorem (for a nice exposition see [12]). One of the consequences is that the spectrum, $M(D)$, of a Douglas algebra $D$ can be identified with a closed subset of $M\left(H^{\infty}+C\right)$.

Note that $M\left(H^{\infty}+C\right)$ itself is $M\left(H^{\infty}\right) \backslash \mathbb{D}$, which is sometimes called the corona of the disk.

Also, $M\left(D_{1}\right)=M\left(D_{2}\right)$ if and only if $D_{1}=D_{2}$. Moreover, the Shilov boundary, $\partial D$, of any Douglas algebra $D$ coincides with $M\left(L^{\infty}(\mathbb{T})\right)$. In particular, $\partial D$ is a proper subset of $M(D)$ for any $D \neq L^{\infty}(\mathbb{T})$. Whereas $L^{\infty}(\mathbb{T})$, the largest Douglas algebra, is a regular algebra, we have the following situation for its proper subalgebras. In what follows, let $k(x)=k_{H^{\infty}+C}(x)$, whenever $x \in M\left(H^{\infty}+C\right)$.

Theorem 3.1. The Shilov boundary, $\partial D$, is the largest set on which a Douglas algebra $D$ is pointwise regular.

This corrects statements in the paper [8] by Daoui, Mahzouli and Zerouali.

Proof. By the remarks that followed the definition of regularity points, we first recall that any regularity point of type one (if it exists) is necessarily contained in $\partial D$. Next we show that $D$, actually, is pointwise regular on $\partial D$. So let $x \in \partial D$ and let $V$ be an open neighborhood of $x$ in $M(D)$. Let $y \in M(D) \backslash V$. Note that $\partial D=M\left(L^{\infty}\right)$. Since by $[15,17] k(y) \cap M\left(L^{\infty}\right)=\{y\}$ whenever $y \in M\left(L^{\infty}\right)$ and $k(y) \cap M\left(L^{\infty}\right)=\emptyset$ whenever $y \in M\left(H^{\infty}+C\right) \backslash M\left(L^{\infty}\right)$, we see that there exists $f_{y} \in H^{\infty}+C \subseteq D$ such that $f_{y}(x)=1$ and $f_{y} \equiv 0$ in a neighborhood (within $M\left(H^{\infty}+C\right)$ ) of $y$. Since $M(D) \backslash V$ is compact, we obtain a function $f=f_{y_{1}} \ldots f_{y_{n}} \in H^{\infty}+C$ with $f(x)=1$ and $f \equiv 0$ on $M(D) \backslash V$. Hence $D$ is pointwise regular on $\partial D$. Thus $\partial D$ is the largest set on which $D$ is pointwise regular.

As an immediate corollary we obtain

Corollary 3.2. Let $D$ be a Douglas algebra. Then the set of hk-continuity points for $D$ is equal to the Shilov boundary, $\partial D$.

In particular, if $D=H^{\infty}+C$, then the set of points of regularity type I coincides with $\partial D$; that is $\mathscr{R}_{I}=\partial D$. It is not known whether in $M\left(H^{\infty}+C\right)$ there are points of regularity type II (see [15, 17] and [21].) Note that this highly contrasts with the situation in $H^{\infty}$, where there are no regularity points of type I and II. 


\section{4. $t$-ANALYTIC SETS}

4.1. General properties of $t$-analytic sets. Throughout this section, $A$ is a Banach function algebra. The following result is immediate from the definition of a $t$-analytic set.

Observation 4.1. Let $E \subseteq M(A)$. Then $E$ is a $t$-analytic set for $A$ if and only if $\bar{E}$ is a t-analytic set for $A$.

Recall that, for $E \subseteq M(A), \hat{E}$ denotes the hull-kernel closure of $E$. It is natural to ask whether the hull-kernel closure of a $t$-analytic set for $A$ is still a $t$-analytic set. The following examples show that this is not necessarily the case.

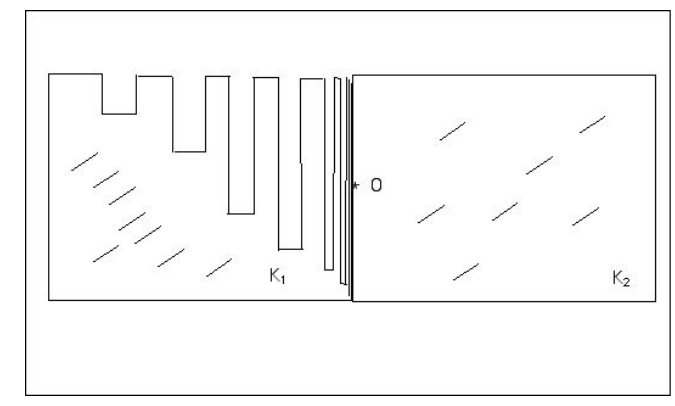

Figure 1. $\hat{K}_{1}=K_{1} \cup K_{2}$

Example 4.2. Let $K_{j}$ be the compact sets in figure 1 and set $K=K_{1} \cup K_{2} \cdot{ }^{1}$ It is well known [28] that the Riemann map $f$ of $K_{1}^{\circ}$ onto the unit disk $\mathbb{D}$ admits a continuous extension to the boundary of $K_{1}$. This extension has constant value at the whole boundary segment to the right of $K_{1}$. Consider the function $g$ defined by $g(z)=f(z)-f(0)$ if $z \in K_{1}$ and $g(z)=0$ if $z \in K_{2}$. Then $g \in A(K)$. Now it is clear that $K_{2}$ and $K_{1}$ are maximal $t$-analytic sets; in particular, $K$ is not $t$-analytic. The hull of the ideal $I_{A(K)}\left(K_{1}\right)$ is $K$. So $K=\hat{K}_{1}$ is the desired set.

The following alternative example, intended for adherents of the glueing technique, is unlike the first, not a point separating algebra on its domain of definition $X$. Recall that $\mathbb{N}=\{0,1,2, \ldots\}$ and $N^{*}=\mathbb{N} \backslash\{0\}$.

Example 4.3. Let $X_{1}$ and $X_{2}$ be two disjoint closed disks in the complex plane and set $X=X_{1} \cup X_{2}$. Choose two convergent sequences of distinct points $a_{n} \in \partial X_{1}$ and $b_{n} \in X_{2}{ }^{\circ}$ such that $b_{0}:=\lim _{n \rightarrow \infty} b_{n} \in X_{2}{ }^{\circ}$. Set $a_{0}=\lim _{n \rightarrow \infty} a_{n} \in \partial X_{1}$. Let

$$
B=\left\{f \in A(X): f\left(a_{n}\right)=f\left(b_{n}\right) \text { for all } n \in \mathbb{N}\right\} .
$$

Then $B$ is a closed sub-algebra of $A(X)$ and $M(A)$ may be identified with the quotient space $X / \sim$ of $X$ obtained by identifying each point $a_{n} \in X_{1}$ with the corresponding point $b_{n} \in X_{2}, n \in \mathbb{N}$. This may also be thought of as glueing together the two disks $X_{1}$ and $X_{2}$ using these two sequences of points.

Let $\pi: X \rightarrow X / \sim$ be the quotient mapping. By Fatou's interpolation theorem $[19$, p. 80$]$ there exists $f \in A\left(X_{1}\right)$ that vanishes exactly at the points $\left\{a_{n}: n \in \mathbb{N}\right\}$.

\footnotetext{
${ }^{1} K_{1}$ appeared in a different setting in [29].
} 
Let $g=f$ on $X_{1}$ and $g=0$ on $X_{2}$. Then $g \in B$. Now as in example 4.2, we see that $\pi\left(X_{j}\right)$ are maximal $t$-analytic sets and the hull of the ideal

$$
I\left(\pi\left(X_{1}\right), X / \sim\right):=\left\{h \in A: h \equiv 0 \text { on } X_{1}\right\}
$$

equals $X / \sim$.

It would be interesting to know for which algebras it is true that the hull-kernel closures of $t$-analytic sets are $t$-analytic.

The following elementary result gives a useful connection between $t$-analytic sets and $k$-hulls of points.

Proposition 4.4. Let $E \subseteq M(A)$ be a t-analytic set for $A$, and suppose that $x \in E$. Then $E \subseteq k_{A}(x)$.

Proof. By the definition of a $t$-analytic set, it is immediate that $J_{A}(x) \subseteq I_{A}(E)$, and hence that $k_{A}(x)=Z\left(J_{A}(x)\right) \supseteq Z_{A}(I(E)) \supseteq E$.

Our next result again shows the strong opposition between $t$-analyticity and regularity.

Proposition 4.5. Let $E \subseteq M(A)$ be a t-analytic subset for $A$ such that $E$ has at least two points. Then $E \cap \mathscr{R}_{I}=\emptyset$ and $E \cap \mathscr{R}_{I I}=\emptyset$. In particular, if $A$ is regular, then the only non-empty, $t$-analytic sets for $A$ are singletons.

Proof. Let $m \in E$. By Proposition 4.4, $E \subseteq k(m)$. Since $E$ has at least two points, $k(m) \neq\{m\}$. Thus $m \notin \mathscr{R}_{I I}$. Hence $E \cap \mathscr{R}_{I I}=\emptyset$.

Now let $x, y \in E$ with $x \neq y$. Suppose, for contradiction, that $x \in \mathscr{R}_{I}$. Let $V$ be a closed neighborhood of $x$ that does not contain $y$. Then, by definition of the set $\mathscr{R}_{I}$, there exists $f \in A$ with $f(x)=1$ and supp $f \subseteq V$. In particular, $f \in J(y)$ and so $f$ vanishes on a non-empty open set that meets $E$. Since $E$ is $t$-analytic, we conclude that $f$ vanishes identically on $E$. This contradicts the choice of $f$. Hence $E \cap \mathscr{R}_{I}=\emptyset$.

The final part of the result now follows from, for example, the fact that $A$ is regular if and only if every point of $M(A)$ is a regularity point of type I (or II).

In $[8$, Proposition 3.5] it was claimed (in our terminology) that no open $t$-analytic set $O$ for $A$ can contain an $h k$-continuity point for $A$. This claim is, in fact, false. For example, if $x$ is an isolated point of some compact space $X$, then $\{x\}$ is an open $t$-analytic set for $C(X)$, but (of course) $x$ is also an $h k$-continuity point for $C(X)$. The following observation corrects this statement, and is now a special case of part of the preceding proposition.

Observation 4.6. Let $O$ be an open t-analytic set for $A$ such that $O$ is not a singleton. Then $O$ does not contain any $h k$-continuity points for $A$.

We now investigate the collection of $t$-analytic sets in more detail. In what follows, the reader should well distinguish between maximal $t$-analytic sets and the maximum $t$-analytic set; the latter being non-existent, in general (see Example 2.4).

Theorem 4.7. Every $t$-analytic set for $A$ is contained in at least one maximal $t$-analytic set for $A$. Moreover, the maximal $t$-analytic sets for $A$ are closed. 
Proof. Let $E$ be a $t$-analytic set for $A$. Consider the (non-empty) collection $\mathcal{F}$ of all $t$-analytic sets for $A$ which contain $E$, partially ordered by inclusion. Let $\left\{Y_{\alpha}: \alpha \in \Lambda\right\}$ be a non-empty chain of sets in $\mathcal{F}$. Then this chain has an upper bound in $\mathcal{F}$; indeed let

$$
Y=\bigcup_{\alpha} Y_{\alpha}
$$

We show that $Y$ is a $t$-analytic set for $A$.

Let $f \in A$ and suppose that $f$ vanishes identically on a non-empty, relatively open subset, $U$, of $Y$. Choose $x_{0} \in U$. Then for all large enough $Y_{\alpha}$, we have $x_{0} \in Y_{\alpha}$. For these $Y_{\alpha}$ we see that $f$ vanishes identically on the non-empty, relatively open subset $U \cap Y_{\alpha}$ of $Y_{\alpha}$, and hence $f$ is constantly 0 on $Y_{\alpha}$. It now follows that $f$ vanishes identically on $Y$, as required.

The existence of the required maximal $t$-analytic sets now follows from Zorn's Lemma. Since the closure of such a maximal $t$-analytic set is still a $t$-analytic set, it is immediate that such maximal $t$-analytic sets must be closed.

It is natural to ask whether each point $x \in M(A)$ is contained in a unique maximal $t$-analytic set. However, this is not generally the case, as is shown by Example 2.4. In that example, both of the sets $X_{1}$ and $X_{2}$ are maximal $t$-analytic sets containing the point 1 .

A description of all $t$-analytic sets appears to be difficult in general. At present, we know such a description only for general regular algebras (Proposition 4.5), and the disk-algebra (Theorem 4.13). Part of the problem is that unions and subsets of $t$-analytic sets need not be $t$-analytic. There is more hope of describing the maximal $t$-analytic sets: we will give some partial results of this type (Theorem 5.12).

Proposition 4.8. Let $E$ be a closed set in $M(A)$ and suppose that $H$ is hull-kernel closed. Then $E \cup H$ is t-analytic if and only if $H \subseteq E$ and $E$ is t-analytic or if $E \subseteq H$ and $H$ is t-analytic.

Proof. The "if" direction is clear. Now suppose that $E \backslash H \neq \emptyset$ and $H \backslash E \neq \emptyset$. Let $y \in E \backslash H$. There exists a function $f \in A$ such that $f \equiv 0$ on $H$, and $f(y) \neq 0$. But $f$ vanishes on the non-empty, relatively open set $(E \cup H) \backslash E=H \backslash E$ in $E \cup H$. Hence $E \cup H$ cannot be $t$-analytic.

Observation 4.9. Suppose that $E_{j}$ are closed sets in $M(A)$ and that $E_{1} \cup E_{2}$ is $t$-analytic. If $E_{1} \nsubseteq E_{2}$, then $E_{2} \subseteq \hat{E}_{1}$.

Proof. We show that $E_{1} \cup E_{2} \subseteq \hat{E}_{1}$. Let $f \in A$ vanish identically on $E_{1}$. Since

$$
U:=\left(E_{1} \cup E_{2}\right) \backslash E_{2}=E_{1} \backslash E_{2} \neq \emptyset,
$$

$f$ vanishes on the non-empty, relatively open subset $U$ of $E_{1} \cup E_{2}$. Hence, the $t$-analyticity implies that $f$ vanishes identically on $E_{1} \cup E_{2}$. Thus $E_{1} \cup E_{2} \subseteq \hat{E}_{1}$, by definition of $\hat{E}_{1}$.

Corollary 4.10. Suppose that $E_{j}$ are closed sets in $M(A)$ and that $E:=E_{1} \cup E_{2}$ is t-analytic. If neither $E_{1}$ is contained in $E_{2}$ nor $E_{2}$ contained in $E_{1}$, then $\hat{E}=$ $\hat{E}_{1}=\hat{E}_{2}$.

Proof. By the observation 4.9 above, $E_{2} \subseteq \hat{E}_{1}$ and $E_{1} \subseteq \hat{E}_{2}$. Since the hullkernel closure of a finite union is the union of the hull-kernel closures, we see that $\hat{E}=\hat{E}_{1}=\hat{E}_{2}$. 
Using Proposition 4.8, we can give certain necessary conditions for a closed set to be $t$-analytic in a Banach function algebra.

Definition 4.1. Let $E$ be a closed subset of a compact Hausdorff space. A nonvoid, proper subset $S$ of $E$ is said to be isolated $^{2}$ (within $E$ ), if there exist two disjoint open sets $U$ and $V$ such that

$$
S \subseteq U \text { and } E \backslash S \subseteq V \text {; }
$$

that is if $S$ can be separated from its complement within $E$.

Corollary 4.11. Let $E \subseteq M(A)$ be a closed $t$-analytic set for $A$. Then either $E$ is a singleton or $E$ does not contain any isolated hull-kernel closed subsets.

Proof. This follows immediately from Proposition 4.8.

The following result is a slight generalization of the previous corollary 4.11.

Proposition 4.12. Let $E \subseteq M(A)$ be a closed $t$-analytic set for $A$. Then for every hull-kernel closed set $H$ in $M(A)$ either $E \backslash H=\emptyset$ or $E \backslash H$ is dense in $E$.

Proof. Suppose, for contradiction, that $\emptyset \neq \overline{E \backslash H} \varsubsetneqq E$. Then there is $y \in E \backslash H$ and $f \in A$ such that $f \equiv 0$ on $H$, but $f(y) \neq 0$. In particular $f \equiv 0$ on the nonvoid (relatively) open set $E \backslash(\overline{E \backslash H}) \subseteq E$. Since $E$ was assumed to be $t$-analytic, we obtain the contradiction that $f(y)=0$.

4.2. $t$-analytic sets in $A(\overline{\mathbb{D}})$. If $A$ is the disk-algebra, then we obtain a complete characterization of the closed $t$-analytic sets. Normalized Lebesgue measure on $\mathbb{T}$ will be denoted by $\sigma$.

Theorem 4.13. Let $E$ be closed subset in $\overline{\mathbb{D}}$. Suppose that $E$ has at least two points. Then $E$ is t-analytic for $A(\overline{\mathbb{D}})$ if and only if $E$ does not contain any isolated

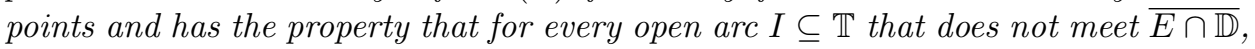
one has that either $I \cap E=\emptyset$ or $\sigma(I \cap E)>0$.

Proof. The necessity (via contraposition) of the condition follows from Corollary 4.11 by using the well known facts ( see [19]) that a closed set $S$ in $\overline{\mathbb{D}}$ is hull-kernel closed if and only if $S$ is the zero set of a function in $A(\overline{\mathbb{D}})$ and that these zero sets are either unions of Blaschke sequences with closed subsets of $\mathbb{T}$ of Lebesgue measure zero or the entire closed disk $\overline{\mathbb{D}}$.

To prove the converse, we first note that if $E \cap \mathbb{D} \neq \emptyset$, then, by our hypotheses, any open set $U$ that meets $E$ within $\mathbb{D}$ has the property that $U \cap E$ is uncountable. Thus, whenever $f$ vanishes on the nonvoid set $U \cap E \cap \mathbb{D}, f$ vanishes everywhere on $\overline{\mathbb{D}}$.

Now if $U$ is an open set in $M(A(\overline{\mathbb{D}}))$ that meets $E \cap \mathbb{T}$, then $U \cap \mathbb{T}$ is a union of pairwise disjoint open $\operatorname{arcs} I_{j}$. Let $f \equiv 0$ on $U \cap E$. Suppose that $I_{j} \cap E \neq \emptyset$

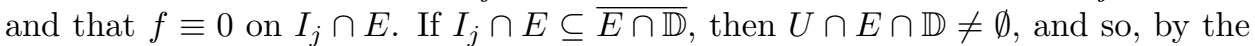
previous paragraph, $f \equiv 0$ on $\overline{\mathbb{D}}$. If $I_{j} \cap E$ is not entirely contained in $\overline{E \cap \mathbb{D}}$, then there exists a subarc $J \subseteq I_{j}$ such that $J \cap \overline{E \cap \mathbb{D}}=\emptyset$ and $J \cap E \neq \emptyset$. In that case, the hypotheses implies that $\sigma(J \cap E)>0$. But $f \equiv 0$ on $J \cap E$. Hence, in this case, too, $f \equiv 0$ on $\overline{\mathbb{D}}$.

To sum up, we have shown that if $f \equiv 0$ on $U \cap E$, then $f$ is the zero function whenever $U \cap E \neq \emptyset$. Hence, $E$ is $t$-analytic.

\footnotetext{
2 or relatively clopen
} 
We deduce the following corollaries.

Corollary 4.14. The only t-analytic hull-kernel closed sets for $A(\overline{\mathbb{D}})$ are the empty set, singletons and the whole spectrum.

Corollary 4.15. Every connected closed set in the spectrum of $A(\overline{\mathbb{D}})$ is t-analytic.

Proof. If $E \subseteq \overline{\mathbb{D}}$ is a singleton, then $E$ is $t$-analytic. So suppose that $E$ contains at least two points. Since $E$ is connected, $E \cap \mathbb{D}$ is either empty, or contains no isolated point. Now if $I \subseteq \mathbb{T}$ is an open arc with $I \cap \overline{E \cap \mathbb{D}}=\emptyset$, then either $I \cap E=\emptyset$ or the connectedness of $E$ implies that $I \cap E$ contains a closed arc. Hence $\sigma(I \cap E)>0$. By Theorem 4.13, $E$ is $t$-analytic for $A(\overline{\mathbb{D}})$.

Corollary 4.16. Every closed set in the spectrum of $A(\overline{\mathbb{D}})$ is componentwise $t$ analytic.

Recall that a uniqueness set for a Banach function algebra $A$ is a nonvoid set $E \subseteq M(A)$ such that any two functions $f$ and $g$ in $A$ that are equal on $E$ already coincide on $M(A)$; in other words if $\left.f\right|_{E}=\left.g\right|_{E}$ implies $f=g$.

Hence we can conclude from Theorem 4.13 that any non-empty $t$-analytic set $E$ for $A(\overline{\mathbb{D}})$ is either a singleton or a uniqueness set for $A(\overline{\mathbb{D}})$.

Such a result is not valid in general; for example in $H^{\infty}$ closures of non-trivial Gleason parts outside $\mathbb{D}$ are $t$-analytic sets (see Section 5 ), but of course not uniqueness sets. On the other hand, the class of uniqueness sets in $A(\overline{\mathbb{D}})$ is much larger than the class of $t$-analytic sets; as examples we may take the set $\left\{1-\frac{1}{n}: n \in \mathbb{N}^{*}\right\}$ (because $\left(1-\frac{1}{n}\right)$ is not a Blaschke sequence), or just $\left\{\frac{1}{n}: n \in \mathbb{N}^{*}\right\}$.

4.3. $t$-analytic sets and prime ideals. The main result of this section is the following surprising relation between $t$-analytic sets and closed prime ideals.

Theorem 4.17. Let $A$ be a Banach function algebra, and let $E \subseteq M(A)$ be a nonvoid t-analytic subset for $A$. Then $I_{A}(E)=\{f \in A: f \equiv 0$ on $E\}$ is a closed prime ideal in $A$.

Proof. Clearly $I_{A}(E)$ is a closed ideal. Let $f$ and $g$ be in $A$ with $f g \in I_{A}(E)$. We show that at least one of $f$ and $g$ is in $I_{A}(E)$. Suppose that $f$ is not in $I_{A}(E)$. Then $U:=E \backslash Z_{A}(f)$ is a nonvoid, relatively open subset of $E$. Since $E \subseteq Z_{A}(f) \cup Z_{A}(g)$, it follows that $g$ is constantly 0 on $U$ and hence, since $E$ is $t$-analytic, $g \in I_{A}(E)$.

Corollary 4.18. Suppose that $E$ is a hull-kernel closed $t$-analytic set for $A$. Then $E$ is connected.

Proof. Since the hull of the closed ideal $I_{A}(E)$ equals $\hat{E}=E$, we see that the quotient algebra $A / I_{A}(E)$ is a Banach algebra with spectrum $E$. The Shilov idempotent theorem and the fact that $I_{A}(E)$ is prime now imply that $E$ must be connected.

\section{5. $t$-ANalyticity in Douglas algebras}

In this section we study the structure of the $t$-analytic sets in Douglas algebras with emphasis on $H^{\infty}+C$. Contrary to the claims in [8], we will see that there is no (non-void) open $t$-analytic set for the smallest Douglas algebra, $H^{\infty}+C$. One of the reasons is that in $H^{\infty}+C$ the $k$-hulls of points are very small sets. To show this, we need a little detour. Since at some points of the proofs, we also need the 
notion of interpolating sequences for Banach function algebras $A$, we recall that notion here.

A sequence $\left(x_{n}\right)$ of points in $M(A)$ is called an $A$-interpolating sequence, if for every bounded sequence, $\left(a_{n}\right)$, of complex numbers there exists $f \in A$ such that $f\left(x_{n}\right)=a_{n}$ for all $n$. In the case of the algebra $H^{\infty}$, the interpolating sequences contained in $\mathbb{D}$ were characterized by L. Carleson $[4,5]$. These are the sequences $\left(z_{n}\right)$ satisfying the condition

$$
\inf _{k \in \mathbb{N}} \prod_{j: j \neq k}\left|\frac{z_{j}-z_{k}}{1-\bar{z}_{j} z_{k}}\right|>0 .
$$

If additionally $\lim _{k \rightarrow \infty} \prod_{j: j \neq k}\left|\frac{z_{j}-z_{k}}{1-\bar{z}_{j} z_{k}}\right|=1$, then one says that $\left(z_{n}\right)$ is a thin sequence. The Blaschke product associated with a thin sequence is called a thin Blaschke product. It is known that any sequence in $\mathbb{D}$ converging to the boundary has a thin subsequence (see [18]).

Recall that, by Carleson's Corona theorem, $\mathbb{D}$ is dense in $M\left(H^{\infty}\right)$. According to Hoffman [20], the set of points $x \in M\left(H^{\infty}+C\right)$ that belong to the closure of an $H^{\infty}$-interpolating sequence in $\mathbb{D}$ is denoted by $G$. The points in $M\left(H^{\infty}+C\right)$ belonging to the closure of thin sequences are called thin points. Both $G$ and the set of thin points are dense in $M\left(H^{\infty}+C\right)$.

Let $Q C=\left\{f \in H^{\infty}+C: \bar{f} \in H^{\infty}+C\right\}$ be the largest $C^{*}$-subalgebra of $H^{\infty}+C$. Let $\rho: M\left(H^{\infty}+C\right) \rightarrow M(Q C)$ be the restriction mapping $\left.m \mapsto m\right|_{Q C}$. It is well known that $\rho$ is a surjection. For each $x \in M(Q C)$, let $\rho^{-1}(x)$ be the QC-level set associated with $x$. These $Q C$-level sets form a partition of $M\left(H^{\infty}+C\right)$ into compact sets. Thus each $m \in M\left(H^{\infty}+C\right)$ is contained in a unique $Q C$-level set. Moreover, a function $f \in H^{\infty}+C$ belongs to $Q C$ if and only if $f$ is constant on the $Q C$-level sets.

The following lemma is surely known to specialists in the field, but we do not know an explicit reference. Our proof depends on Hoffman's theory of the structure of the maximal ideal space of $H^{\infty}$ and on Wolff's interpolation theory for $Q C \cap H^{\infty}$ functions.

Lemma 5.1. The $Q C$-level sets are nowhere dense in $M\left(H^{\infty}+C\right)$.

Proof. Suppose, for contradiction, that some $Q C$-level set has nonempty interior $U$ in $M\left(H^{\infty}+C\right)$. Let $x \in U$. We first prove (by a purely topological reasoning) the existence of an open set $O$ in $M\left(H^{\infty}\right)$ such that $x \in \bar{O} \cap M\left(H^{\infty}+C\right) \subseteq U$. To this end, let $V$ be an open set in $M\left(H^{\infty}+C\right)$ such that $x \in V \subseteq \bar{V} \subseteq U$. Since $M\left(H^{\infty}\right)$ is a normal space, we can separate the compact sets $M\left(H^{\infty}+C\right) \backslash U$ and $\bar{V}$ by two open sets in $M\left(H^{\infty}\right)$. Let us call the open set containing $\bar{V}$ by $O$. It is now clear that $x \in \bar{O} \cap M\left(H^{\infty}+C\right) \subseteq U$.

Since $G$ is dense in $M\left(H^{\infty}+C\right)$, there is $y \in G \cap\left(O \cap M\left(H^{\infty}+C\right)\right)=G \cap O$. By definition of $G$, there exists an $H^{\infty}$-interpolating sequence $\left(z_{n}\right)$ in $O \cap \mathbb{D}$ such that $y \in \overline{\left\{z_{n}: n \in \mathbb{N}\right\}}$. Thus

$$
\overline{\left\{z_{n}: n \in \mathbb{N}\right\}} \cap M\left(H^{\infty}+C\right) \subseteq \bar{O} \cap M\left(H^{\infty}+C\right) \subseteq U .
$$

Let $\left(w_{n}\right)$ be a thin subsequence of $\left(z_{n}\right)$. Then by [33], $\left(w_{n}\right)$ is an interpolating sequence for functions in $Q C \cap H^{\infty}$. In particular, $\left(w_{n}\right)$ has at most a single cluster point in each $Q C$-level set. Since the set of cluster points is infinite, we get a contradiction to our assumption that $U$ is contained in a single $Q C$-level set. 
Lemma 5.2. The $k$-hulls $k(x)$ of points in $M\left(H^{\infty}+C\right)$ are nowhere dense.

Proof. Since $Q C$ is isometrically isomorphic to $C(M(Q C)), Q C$ is a regular subalgebra of $H^{\infty}+C$. By Proposition 2.1 the $k$-hulls of points in $M(Q C)$ are singletons. Now it is clear that for any $m \in M\left(H^{\infty}+C\right)$ the $k$-hull $k(m)$ is contained in the unique $Q C$-level set $E_{m}$ containing $m$. Since, by Lemma 5.1, $E_{m}$ is nowhere dense in $M\left(H^{\infty}+C\right)$, the same is true for its subsets; in particular for $k(m)$.

Theorem 5.3. There is no non-empty, open t-analytic set in $M\left(H^{\infty}+C\right)$.

Proof. Suppose that $O$ is a $t$-analytic open subset of $M\left(H^{\infty}+C\right)$. Let $x \in O$. By Proposition 4.4, $O \subseteq k(x)$. Since by Lemma 5.2, $k(x)$ is nowhere dense, we see that $k(x)$ has no interior points. Thus $O=\emptyset$.

On the other hand, there do exist Douglas algebras that are $O$-analytic for some open sets. To present an example, we need some further facts and notions from Hoffman's theory.

One of those facts tells us that every $m \in M\left(H^{\infty}\right)$ has a unique representing measure on the Shilov boundary of $H^{\infty}$. The associated support of that measure is denoted by supp $m$. Note that the Shilov boundary of $H^{\infty}$ is identified with $M\left(L^{\infty}\right)$.

Recall that a Gleason part of a uniform algebra is a set of the form

$$
P(m)=\{x \in M(A): \rho(x, m)<1\},
$$

where $\rho(x, m)=\sup \left\{|\hat{f}(x)|:\|\hat{f}\|_{\infty} \leq 1, \hat{f}(m)=0\right\}$ is the pseudo-hyperbolic distance. The Gleason parts for $H^{\infty}$, and hence for any Douglas algebra, were characterized by Hoffman ([20]). It turned out that they can be divided into two classes: the trivial parts (these are those for which $P(m)$ is the singleton $\{m\}$,) and the nontrivial parts. Hoffman showed that $P(m)$ is non-trivial if and only if $m$ belongs to the closure of an $H^{\infty}$-interpolating sequence, that is $m \in G$. For example, every point of the Shilov boundary of $H^{\infty}$ has a trivial Gleason part. Also, for every $m \in M\left(H^{\infty}\right)$ there exists a continuous map $L_{m}$ of $\mathbb{D}$ onto $P(m)$ such that $f \circ L_{m}$ is analytic for all $f \in H^{\infty}$. Here $L_{m}$ is a bijection if and only if $P(m)$ is non trivial, and $L_{m}$ is constant otherwise. Due to the Chang-Marshall theory, it is known that the Gleason parts $P(x)$ for Douglas algebras coincide with those for $H^{\infty}$ whenever $x \in M(D)$.

The next proposition follows immediately from the above facts concerning analytic structure in the character space.

Proposition 5.4. Let $D$ be a Douglas algebra (or $D=H^{\infty}$ ) and let $m \in M(D)$. Then $P(m)$ and $\overline{P(m)}$ are $t$-analytic sets.

The following follows from standard arguments in the theory of Douglas algebras (see [12] and [11]).

Example 5.5. Let $m$ be a thin point in $M\left(H^{\infty}+C\right)$, and consider the Douglas algebra

$$
D=\left\{f \in L^{\infty}:\left.f\right|_{\operatorname{supp} m} \in H^{\infty} \mid \operatorname{supp} m\right\} .
$$

Then $M(D)=M\left(L^{\infty}\right) \cup\left\{x \in M\left(H^{\infty}\right): \operatorname{supp} x \subseteq \operatorname{supp} m\right\}$. By [3], the thin Blaschke product $b$ associated with $m$ vanishes only at $m$, when looked upon as an element in $D$. Moreover $O:=P(m)=\{x \in M(D):|b(x)|<1\}$ is open in $M(D)$. It is evident that $O$ is $t$-analytic. 
In view of Proposition 5.4 and the fact that we were unable to find other examples, we ask the following question.

Q1 Let $D$ be a Douglas algebra. Is every maximal $t$-analytic set for $D$ the closure of a Gleason part?

In the following we give some results supporting this conjecture. It is also closely related to Alling's conjecture (see Section 6).

Note that if $E \subseteq M(D)$ is a $t$-analytic set for $D$, then it is $t$-analytic for $H^{\infty}+C$.

Lemma 5.6. Let $D$ be a Douglas algebra and $x \in M(D)$. Then $k_{D}(x) \subseteq k_{H^{\infty}+C}(x)$.

Proof. This follows from the definition that $k_{D}(x)$ is the intersection of the zero sets of all functions in $D$ that vanish in neighborhood (within $M(D)$ ) of $x$ and the fact that $H^{\infty}+C \subseteq D$.

In what follows we use again the symbol $k(x)$ for $k_{H^{\infty}+C}(x)$.

Proposition 5.7. Let $x$ be a point in the Shilov boundary $\partial D$ of a Douglas algebra $D$. Then $\{x\}$ is a maximal t-analytic set for $D$.

Proof. Let $E$ be a $t$-analytic set for $D$ containing $x$. By Lemma 4.4, we have that $E \subseteq k_{D}(x)$. By $[15,17] k(x) \cap M\left(L^{\infty}\right)=\{x\}$; hence we see from Lemma 5.6 above that $k_{D}(x) \cap \partial D=\{x\}$. Now if $y \in E \backslash \partial D$, then, by [15], $k(y) \cap M\left(L^{\infty}\right)=\emptyset$. Thus there exists $f \in H^{\infty}+C \subseteq D$ that vanishes in a neighborhood (within $M\left(H^{\infty}+C\right)$ ) of $y$, but not at $x$. This contradicts the $t$-analyticity of $E$. Thus $E=\{x\}$.

Proposition 5.8. Let $E$ be a non-empty, hull-kernel closed subset of the set $X$ of trivial points in $M\left(H^{\infty}+C\right)$. Then $E$ is t-analytic if and only if $E$ is a singleton.

Proof. The "if" part is trivial. Now suppose that $E$ is $t$-analytic. By Corollary 4.18, $E$ must be connected. However, by [30], the set $X$ is totally disconnected. Hence $E$ is a singleton.

Observation 5.9. Let $E \subseteq M\left(H^{\infty}+C\right)$ be a t-analytic set for $H^{\infty}+C$. Suppose that $x$ and $y$ are two points in $E$. Then $k(x)=k(y)$.

Proof. By Lemma 4.4, $E \subseteq k(x) \cap k(y)$. Suppose, for contradiction, that $x \notin k(y)$. Then there exist $f \in J(y)$ such that $f(x) \neq 0$. Since $f$ is non-constant on $E$, but $f$ vanishes identically on a non-empty, relatively open set in $E$ we see that $E$ cannot be a $t$-analytic subset of $M\left(H^{\infty}+C\right)$. We conclude that $x \in k(y)$ and so, by [17], $k(x) \subseteq k(y)$. Interchanging the role of $x$ and $y$ yields that $k(x)=k(y)$.

In analogy to the observation 5.9 above, we have a similar situation whenever $E$ is the hull of a closed prime ideal (see also question Q3 in the next section).

Observation 5.10. Let $I$ be a closed prime ideal in $H^{\infty}+C$. Then $k(x)=k(y)$ whenever $x, y \in Z(I)$.

Proof. By [16, Theorem 1.9], $Z(I) \subseteq k(x) \cap k(y)$. Suppose, for contradiction, that $y \notin k(x)$. Then there exists $f \in J(x)$ with $f(y) \neq 0$. But by [16, Corollary 1.11] $J(x) \subseteq I$; thus $f \in I$ and so, since $y \in Z(I), f(y)=0$. This is a contradiction. Hence $y \in k(x)$. Thus, by [17], $k(y) \subseteq k(x)$. Since the argument is symmetric in $x$ and $y$, we obtain the assertion that $k(x)=k(y)$.

We can now strengthen the assertion of the observation 5.9. 
Corollary 5.11. Let $E$ be a t-analytic set for $H^{\infty}+C$. Then $k(x)=k(y)$ for each $x, y \in \hat{E}$.

Proof. This follows from Theorem 4.17 that $I_{H^{\infty}+C}(E)$ is a prime ideal and observation 5.10 .

We note that, for general function algebras, this assertion is no longer true. In fact, let $A=A(K)$ be the example 4.2. Then $k(x)=K_{1} \cup K_{2}$ for every $x \in K_{1}$, where $K_{1}$ is a maximal $t$-analytic set, but $k(y)=K_{2}$ for $y \in K_{2}^{\circ}$. Although $y$ belongs to the hull of $I_{A}\left(K_{1}\right), k(y)$ is strictly smaller than $k(x)$.

We now come to the main (and concluding) result of this section. In what follows, let $\{|f|<1\}=\left\{x \in M\left(H^{\infty}+C\right):|f(x)|<1\right\}$ whenever $f \in H^{\infty}+C$.

Theorem 5.12. Let $E$ be a maximal t-analytic set for $H^{\infty}+C$ containing a thin point $x \in M\left(H^{\infty}+C\right)$. Then $E=\overline{P(x)}$.

Proof. By Lemma 4.4, we know that $E \subseteq k(x)$. Let $b$ be a thin Blaschke product with $b(x)=0$. Let $Q(x)$ be the $Q C$-level set containing $x$. We know that $k(x) \subseteq$ $Q(x)$ (see the proof of Lemma 5.2 or [15]) and that $\{|b|<1\} \cap Q(x)=P(x)$ (see [21]). Thus $\{|b|<1\} \cap k(x)=P(x)$. Moreover, $\overline{P(x)}$ is hull-kernel closed (see [13]). Two cases now appear.

Case 1 If $k(x) \subseteq \overline{P(x)}$, then it easily follows that $k(x)=\overline{P(x)}$. Hence, by the maximality of $E$ and Proposition 5.4,E $=\overline{P(x)}$. (Note this case can actually happen, see [16]).

Case 2 If $k(x) \nsubseteq \overline{P(x)}$, let $y \in k(x) \backslash \overline{P(x)}$. Then there exists $f \in H^{\infty}$ such that $f \equiv 0$ on $\overline{P(x)}$, but $f(y) \neq 0$. Thus the zero set of $f$ meets $E$ in a relatively open set, namely $\{|b|<1\} \cap E$, but $f(y) \neq 0$. Since $E$ is assumed to be $t$-analytic, we conclude that $y \notin E$. Thus $E \subseteq \overline{P(x)}$. Proposition 5.4 and the maximality of $E$ now imply that $E=\overline{P(x)}$.

We observe that by [21], $k(x)$ is a minimal $k$-hull for every $x \in Z(b)$ whenever $b$ is a thin Blaschke product and that $P(x)$ is a maximal Gleason part with maximal closure (see [3]).

\section{Is there A COUNTER-EXAmple to Alling's CONJECTURe?}

Alling's famous conjecture [1] reads as follows: Let $I$ be a non-maximal closed prime ideal in $H^{\infty}$. Does there exist a point $x \in G$ such that

$$
I=\left\{f \in H^{\infty}: f \equiv 0 \text { on } \overline{P(x)}\right\} ?
$$

(See also [25]). It is easy to see that every such ideal actually is prime. In [14], it was shown that if $I$ is a non-maximal closed prime ideal in $H^{\infty}$ then $I=I_{H^{\infty}}(E)$ for some hull-kernel closed subset $E \in M\left(H^{\infty}+C\right)$. The analogous result is true for prime ideals in $H^{\infty}+C$; see [16]. So Alling's conjecture reduces to the problem of determining the hull of $I$ : Is $Z_{A}(I)=\overline{P(x)}$ for every non-maximal closed prime ideal $I$ in $A=H^{\infty}$ or $H^{\infty}+C$ ?

Theorem 4.17 gives us another way to construct closed prime ideals. It says that if $E$ is a $t$-analytic set for $A$, then $I_{A}(E)$ is prime.

Thus every hull-kernel closed $t$-analytic set $E$ for $H^{\infty}+C$ that is not a singleton yields a non maximal closed prime ideal. This raises the following question: 
Q2 Let $E \subseteq M\left(H^{\infty}+C\right)$ be a hull-kernel closed $t$-analytic set for $H^{\infty}+C$. Suppose that $E$ is not a singleton. Is $E$ equal to $\overline{P(x)}$ for some $x \in G$ ?

Unfortunately this does not prove Alling's conjecture for prime ideals containing a thin point in their hull since we do not know whether the hull of such an ideal is a $t$-analytic set. This raises the next question.

Q3 Is the hull of every closed prime ideal in $H^{\infty}+C$ a $t$-analytic set?

Example 4.2 shows that it is not always true that the hull of a closed prime ideal in an arbitrary Banch function algebra is a $t$-analytic set. In that example the zero ideal is prime, and the hull of the zero ideal is $M(A)$, which is not $t$-analytic.

Note that, if questions Q2 and Q3 have a positive answer, then Alling's conjecture is true.

Finally, we mention that a class of closed prime ideals given by Suárez [31] is a special case of our class whose construction can be done using $t$-analytic sets. In fact, fix $y \in G$. Consider the following system of $t$-analytic sets:

$$
\mathfrak{S}:=\{\overline{P(x)}: y \in \overline{P(x)}\}
$$

and let $E=\bigcup_{M \in \mathfrak{S}} M$. Suárez [31] showed that the ideal $I_{H^{\infty}}(M)$ is a closed prime ideal. But $\mathfrak{S}$ is a chain (see [31]); so we have that $E$ actually is $t$-analytic. So his result is a consequence of our Theorem 4.17.

\section{Acknowledgements}

Part of this paper was created during a visit of the first author at the Université Paul Verlaine, Metz, in 2004. He thanks the LMAM for its generous support. The authors would also like to thank Norbert Steinmetz for confirming that the Riemann map in Example 4.2 does admit a continuous extension as claimed.

\section{REFERENCES}

[1] N. Alling, Aufgabe 2.3, Jahresbericht Deutsch. Math. Verein. 73 (1971/72), no. 2, p. 2.

[2] A. Browder, Introduction to Function Algebras, W.A. Benjamin, Inc. New York, 1969.

[3] P. Budde, Support sets and Gleason parts, Michigan Math. J. 37 (1990), 367-383.

[4] L. Carleson, An interpolation problem for bounded analytic functions, Amer. J. Math. 80 (1958), 921-930.

[5] L. Carleson, Interpolations by bounded analytic functions and the corona problem, Ann. of Math. 76 (1962), 547-559.

[6] I. Colojoară, C. Foiaş, Theory of generalized spectral operators, Mathematics and its Applications, Vol. 9. Gordon and Breach, Science Publishers, New York-London-Paris, 1968.

[7] H. G. Dales, Banach algebras and automatic continuity, London Mathematical Society Monographs. New Series, vol. 24, The Clarendon Press Oxford University Press, New York, 2000, Oxford Science Publications.

[8] A. Daoui, H. Mahzouli, E. Zerouali, Sur les algèbres $S$-régulières et la $S$-décomposabilité des opérateurs de multiplication, Proc. Amer. Math. Soc. 131 (2003), 3211-3220.

[9] J.F. Feinstein, D. Somerset, Non-regularity for Banach function algebras, Studia Math. 141 (2000), 53-68.

[10] Şt. Frunză, A characterization of regular Banach algebras, Rev. Roumaine Math. Pures Appl. 18 (1973), 1057-1059.

[11] T. Gamelin, Uniform Algebras, Second Edition, Chelsea, New York, 1984.

[12] J.B. Garnett, Bounded analytic functions, Pure and Applied mathematics, 96. Academic Press, New York-London, 1981. xvi+467

[13] P. Gorkin, Gleason parts and COP, J. Funct. Anal. 83 (1988), 44-49.

[14] P. Gorkin, R. Mortini, Alling's conjecture on closed prime ideals in $H^{\infty}$, J. Funct. Anal. 148 (1997), 185-190. 
[15] P. Gorkin, R. Mortini, Synthesis sets for $H^{\infty}+C$, Indiana Univ. Math. 49 (2000), 287-309.

[16] P. Gorkin, R. Mortini, Hulls of closed prime ideals in $H^{\infty}$, Illinois J. Math. 46 (2002), 519532.

[17] P. Gorkin, R. Mortini, $k$-hulls and support sets in the spectrum of $H^{\infty}+C$, Archiv Math. 79 (2002), 51-60.

[18] P. Gorkin, R. Mortini, Universal Blaschke products, Math. Proc. Camb. Phil. Soc. 136 (2004), $175-184$

[19] K. Hoffman, Banach spaces of analytic functions. Reprint of the 1962 original. Dover Publications, Inc., New York, 1988.

[20] K. Hoffman, Bounded analytic functions and Gleason parts, Ann. of Math. 86 (1967), $74-111$.

[21] K. Izuchi, $K$-hulls of $Q C$-level sets, Indiana Univ. Math. 52 (2003), 421-436.

[22] K. Izuchi, Y. Izuchi, On a class of closed prime ideals in $H^{\infty}$, Complex Var. 50 (2005), 1011-1023.

[23] K. Laursen, M. Neumann, An introduction to local spectral theory, Oxford Sci. Publ. 2000.

[24] G. Leibowitz, Lectures on Complex Function Algebras, Scott, Foresman and Company, 1970.

[25] R. Mortini, Gleason parts and prime ideals in $H^{\infty}$, Linear and complex analysis problem book 3, Part I (V.P. Havin, N.K. Nikolski, eds.), Lecture Notes in Math. vol 1573, Springer Verlag, Berlin, 1994, pp. 136-138.

[26] R. Mortini, Decomposable multiplication operators on $H^{\infty}+C$, Archiv Math. 72 (1999), 64-67.

[27] M. Neumann, Commutative Banach algebras and decomposable operators, Monatsh. Math. 113 (1992), 227-243.

[28] Ch. Pommerenke, Boundary behaviour of conformal maps, Springer-Verlag, Berlin, 1992.

[29] R. Rupp, Stable rank and boundary principle, Topology Appl. 40 (1991), 307-316.

[30] D. Suárez, Trivial Gleason parts and the topological stable rank of $H^{\infty}$, American J. Math. 118 (1996), 879-904.

[31] D. Suárez, Maximal Gleason parts for $H^{\infty}$, Michigan Math. J. 45 (1998), 55-72.

[32] C. Sundberg, T.H. Wolff, Interpolating sequences for $Q A_{B}$, Trans. Amer. Math. Soc. 276 (1983), 551-581.

[33] T.H. Wolff, Some theorems on vanishing mean oscillation, Ph.D. Thesis, Univ. of California, Berkeley, 1979.

[34] T.H. Wolff, Two algebras of bounded functions, Duke Math. J. 49 (1982), 321-328.

School of Mathematical Sciences, University of Nottingham, University Park, NotTingham NG7 2RD, UK

E-mail address: Joel.Feinstein@nottingham.ac.uk

Département de Mathématiques, LMAM, UMR 7122, Université Paul Verlaine, Ile du Saulcy, F-57045 Metz, France

E-mail address: mortini@univ-metz.fr 